\title{
Low power thrust measurements of the water electrolysis Hall effect thruster
}

\author{
Alexander Schwertheim ${ }^{1}\left[\right.$ ] Aaron Knoll ${ }^{1}[$
}

Received: 19 October 2020 / Revised: 18 December 2020 / Accepted: 14 January 2021 / Published online: 1 March 2021

(c) The Author(s) 2021

\begin{abstract}
We propose that a Hall effect thruster could be modified to operate on the products of water electrolysis. Such a thruster would exploit the low cost and high storability of water while producing gaseous hydrogen and oxygen in-situ as they are required. By supplying the anode with oxygen and the cathode with hydrogen, the poisoning of the cathode is mitigated. The water electrolysis Hall effect thruster (WET-HET) has been designed to demonstrate this concept. The dimensions of the WET-HET have been optimized for oxygen operation using PlasmaSim, a zero-dimensional particle in cell code. We present the first direct thrust measurements of the WET-HET. A hanging pendulum style thrust balance is used to measure the thrust of the WET-HET while operating in the Boltzmann vacuum facility within the Imperial Plasma Propulsion Laboratory. For this test the beam was neutralized using a filament plasma bridge neutralizer operating on krypton. We find thrust, specific impulse, and thrust efficiency all increase linearly with power for values between 400 and $1050 \mathrm{~W}$. Increasing the mass flow rate from 0.96 to $1.85 \mathrm{mg} / \mathrm{s}$ increases thrust at the expense of specific impulse. Changing mass flow rate was found to have little impact on the thrust efficiency over this range. An optimal radial magnetic flux density of $403 \mathrm{G}$ at the exit plane is found. Further increases to the magnetic field beyond this point were found to decrease the thrust, specific impulse and thrust efficiency, whereas the discharge voltage increased monotonically with increasing magnetic field for a given input power. It was found that the experimental thruster performance was lower than the simulation results from PlasmaSim. However, the general trends in performance as a function of power and propellant mass flow rate were preserved. We attribute a portion of this discrepancy to the inability of the simulation to model the energy absorbed by the covalent bond of the oxygen molecule. For the powers and mass flow rates surveyed we measured thrust ranging from $4.52 \pm 0.18$ to $8.45 \pm 0.18 \mathrm{mN}$, specific impulse between $324 \pm 12$ and $593 \pm 12 \mathrm{~s}$, and anode thrust efficiencies between $1.34 \pm 0.10$ and $2.34 \pm 0.10 \%$.
\end{abstract}

Keywords Hall effect thruster $\cdot$ Alternative propellants $\cdot$ Water propulsion $\cdot$ Electrolysis $\cdot$ Multimode propulsion

\section{Introduction}

Xenon has dominated the market as the de-facto standard propellant for both Hall effect thrusters (HETs) and gridded ion engines (GIEs) since the commercialization of electric propulsion (EP) technologies [12]. The high and volatile price of xenon has driven many to search for alternative propellants to supply the growing demand for less expensive EP systems. Krypton has been identified by many as the most

Alexander Schwertheim

a.schwertheim18@imperial.ac.uk

Aaron Knoll

a.knoll@imperial.ac.uk

1 Imperial College London, South Kensington, London, UK likely substitute to xenon. It shares the same chemical inertness as xenon, which is crucial for compatibility with the low work function thermionic emitters within the cathodes used in EP missions [18]. Krypton has shown promising results in the laboratory, demonstrating a greater specific impulse $\left(I_{\mathrm{sp}}\right)$ at a considerably lower cost than xenon [13]. The major hurdle standing in the way of broad krypton adoption is the significantly lower density when stored in typical conditions: $0.53 \mathrm{~g} / \mathrm{cc}$ for krypton compared to $1.6 \mathrm{~g} / \mathrm{cc}$ for xenon at $50{ }^{\circ} \mathrm{C}$ and $14 \mathrm{Mpa}$ [26]. The limitation of storage density appears to be restricting krypton use to low delta-V missions or those with very large propellant tanks.

Iodine has recently enjoyed attention as a promising electric propellant. Iodine can be stored as a solid at greater densities than xenon, and has a similar electron-impact cross section [12]. The main caveat of iodine is the fact that it will 
strongly corrode many of the materials commonly found on spacecraft [14].

We suggest that with some modification a HET could effectively use water as a propellant through the utilization of an electrolyzers. We refer to such a system as a water electrolysis HET. Water is stored as a liquid and converted into gaseous oxygen and hydrogen in situ as they are consumed. This eliminates the need for high pressure storage tanks altogether. Unlike all other proposed propellants, water is extremely cheap, non-toxic and can be easily stored at a moderate density at low pressure. Furthermore, the ubiquity of water throughout the solar system opens the possibility to future in situ resource utilization (ISRU) opportunities [29]. We suggest that the anode of the HET be operated on the oxygen produced, with the accompanying cathode only utilizing the hydrogen.

Switching the operating gas in a HET from xenon to oxygen results in a decrease in the ion molar mass by a factor of 4.09, suggesting a higher $I_{\text {sp }}$ can be expected yet at a considerably lower thrust [22]. When a sufficiently energetic electrons collides with a xenon atom, the atom will either be electrically excited, ionized, or both. In the context of a HET the energy expended to the electrical excitation of the atom does not contribute to thrust, and is considered lost for the purpose of generating thrust. Oxygen is a diatomic molecule where xenon is monatomic. Much like xenon an oxygen molecule can become electrically excited, or ionized, yet here the energy of the electron can also be converted into rotational and vibrational energy of the covalent bond, which we again consider to be a loss. An electron collision also has the ability to dissociate an oxygen molecule, creating two atoms which can themselves be ionized to $\mathrm{O}^{+}$ions [22]. These additional energy loss mechanisms are predicted to reduce the thrust efficiency of a water electrolysis HET, yet quantitatively estimating such a reduction is difficult.

A vital component of a HET propulsion system is the electron source referred to as the neutralizer or cathode. The most common cathodes used in EP missions are of the hollow cathodes type. The emitters in these cathodes are typically either lanthanum hexaboride $\left(\mathrm{LaB}_{6}\right)$ or barium oxide impregnated in porous tungsten [9]. Hollow cathodes operate by heating the emitter to high temperatures to promote thermionic electron emission from the low work function material. By subjecting the emitter to an electric potential and an inert working gas, an electron current can be extracted from the emitter surface. In a typical xenon EP missions the cathode operates on the same xenon supply as the thruster anode but at approximately $10 \%$ of the anode mass flow rate. The heated emitters are extremely sensitive to impurities in the working gas. Even trace amounts of reactive gases such as air, oxygen or water can "poison" the emitter, drastically reducing the electron current that can be extracted $[2,7]$. The threat of poisoning these mission critical cathodes dictates that only the highest purity xenon or krypton has been flown. Furthermore, the high chemical sensitivity of the cathodes emitters greatly restricts the search for alternative propellants to all but the purist of inert gases. Incompatibilities with traditional EP cathodes and iodine for example are driving research into new cathode emitters [27].

Water electrolysis produces $89 \%$ oxygen and $11 \%$ hydrogen by mass. We have identified that for a water electrolysis HET this ratio can be exploited as a coincidentally convenient anode-to-cathode mass flow ratio. This means that the cathode is operated solely on pure hydrogen. Hydrogen is one the few non-noble gases which has shown to be highly compatible with $\mathrm{LaB}_{6}$ cathodes when tested in the laboratory $[8,25]$. Gallagher measured how aggressively different gases poison $\mathrm{LaB}_{6}$ cathodes [7]. Gallagher found that not only did hydrogen not poison the cathode, but the gas appears to reverse any poisoning of the emitters, in a process which was assumed to be hydrogen-ion bombardment [7]. This suggests that even if the anode of a water electrolysis HET is operated on the "poisonous" oxygen, the cathode would be able to operate unaffected on the produced hydrogen. A cathode mass flow rate of $10 \%$ compared to the anode flow rate has been shown to be ideal for traditional xenon-fueled HETs. Whether this ratio holds for an oxygen/hydrogen HET must be tested experimentally.

The electrolysis of water in space is already an established and proven technology used for human space flight applications within the life support equipment, for instance on the International Space Station [5]. A water electrolysis HET must expend energy to split water into its constituent elements. A traditional HET such as an SPT-100 operates at $1.35 \mathrm{~kW}$ on $50 \mathrm{sccm}$ of xenon, with a further $5 \mathrm{sccm}$ of xenon supplying the cathode [21]. To generate a comparable volume of propellant, an electrolyser with an $80 \%$ efficiency requires $30 \mathrm{~W}$ to convert $1.5 \mathrm{mg} / \mathrm{s}$ of water into $56 \mathrm{sccm}$ of oxygen and $111 \mathrm{sccm}$ of hydrogen [16]. The additional power expended for electrolysis is less than $3 \%$ of the discharge power. The mass penalty incurred by including a small electrolyser will be offset to some extent by a low-pressure water tank replacing traditional pressurized xenon tanks. An interesting side-effect is that hydrogen is the highest performing propellant for electrothermal propulsion systems such as resistojets [15]. If we assume the hydrogen in the above example to be heated to $1000 \mathrm{~K}$ by the cathode, the thermal expansion of this gas will generate an additional $1 \mathrm{mN}$ of additional thrust for a forward facing cathode.

The chemical propulsion community has identified in situ water electrolysis as a highly storable, green and safe method of benefiting from the high performance of a hydrogen-oxygen bi-propellant engine for in-space propulsion [16]. Liquid water counterintuitively presents a higher hydrogen storage density than even cryogenically cooled liquid hydrogen: a 
liter of water contains $111 \mathrm{~g}$ of hydrogen, where a liter of liquid hydrogen only weighs $90 \mathrm{~g}$. Water electrolysis chemical propulsion has performed very well in ground testing, with several systems expected to fly in the near future $[4,11,16]$. An exciting opportunity exists to pair a chemical water electrolysis propulsion system with a water electrolysis HET on a single spacecraft. By sharing hardware such as a propellant tank, electrolyser and propellant management system, the mass penalty of flying two propulsion systems is dramatically reduced. Such a multimode propulsion system has the ability to perform high thrust maneuvers such as Hohmann transfers, orbital insertions, and proximity flying using the chemical mode, but then also make high specific impulse burns for electric orbit raising, station keeping and interplanetary cruises [23]. Shared propellant multimode propulsion systems such as this can enjoy an unmatched degree of mission flexibility and adaptability even after launch [20].

\section{The water electrolysis Hall effect thruster}

An oxygen molecule has a similar first energy of ionization to xenon at $12.07 \mathrm{eV}$ and $12.13 \mathrm{eV}$ respectively, yet an oxygen molecule has a considerably smaller electronimpact ionization cross section [10, 24]. This discrepancy suggests that we should expect an oxygen plasma to behave very differently to a xenon plasma at a similar temperature. By extension we expect that a thruster optimized for xenon will be different to a thruster optimized for oxygen.

The water electrolysis Hall-effect thruster (WET-HET) has been constructed to experimentally test the feasibility of using water electrolysis as a HET propellant. This prototype has been designed to operate like a traditional xenon HET, yet with the channel dimensions and magnetic topology optimized for oxygen operation. The annular internal channel dimensions of a HET determine the volume-to-surface ratio of the plasma and the gas residence time. For the WET-HET these channel dimensions were optimize for oxygen using an in-house particle in cell (PIC) model called PlasmaSim [23]. This code is a fully kinetic three dimensional simulation using a Boris pusher model. Collisions are calculated using direct simulation Monte Carlo, using the electron-impact cross sections form Itikawa and Stephan et al. [10, 24].

Designing a thurster to operate on such a novel propellant required us to perform a sensitivity study with PlasmaSim across the following parameters: mass flow rate, anode potential, channel width, channel length and channel circumference. Fully kinetic PIC codes such as PlasmaSim are computationally demanding, and required hundreds of $\mathrm{CPU}$ hours for even 1-dimensional solutions at realistic number densities and geometric scales. We chose instead to simplify PlasmaSim to a quasi-zero dimensional version of PlasmaSim, which we refer to as PlasmaSim 0D. This version tracks all particles through three dimensions, yet reduces the simulation domain to a single cell which represents the annular thruster channel. We impose a constant electric field in the axial direction, and do not simulate a magnetic field at all. Instead, the electrons have a temperature described by a Boltzmann distribution centered about a temperature we specify. This electron temperature is the spatial average for the entire channel, which is very different from a real thruster, where the electron temperature varies greatly along the axis [1]. This makes choosing a single electron temperature as the spatial average temperature difficult. The PlasmaSim 0D code has been validated against experimental data for an SPT-100 thruster operating on xenon [23]. A sensitivity analysis was performed to find which electron temperature best replicated the ionization fraction and exit velocity of the SPT-100. This was found to be $11.5 \mathrm{eV}$, which falls within the mean electron energy range of 10-20 $\mathrm{eV}$ found experimentally [3]. Given the novelty of oxygen as a HET propellant, it is very difficult to predict what a representative electron temperature should be. For this reason, we again select $11.5 \mathrm{eV}$ as the electron temperature when simulating oxygen, with the intention of using a more representative number once it can be determined experimentally. Additionally, the sensitivity of the results to the assumed electron temperature have been evaluated below. For the ions, we have assumed a temperature of $0.1 \mathrm{eV}$ to conform with other PIC models [6].

PlasmaSim 0D can perform a simulation in less than half an hour, allowing us to optimize the thruster by quickly simulating many different set-points and geometries. This version of PlasmaSim has been greatly simplified, yet a sensitivity analysis of dimensions and mass flow rate of a real thruster have shown promising results in Schwertheim et al. [23]. Assuming an electron temperature of $11.5 \mathrm{eV}$, the final optimized dimensions and operational point for the WETHET are as follows:

- Channel width: $5 \mathrm{~mm}$.

- Channel depth: 35-60 mm.

- Channel outer diameter: $25 \mathrm{~mm}$.

- Mass flow rate: $1-2 \mathrm{mg} / \mathrm{s}(40-80 \mathrm{sccm})$.

- Power: 1-2 kW.

The thermal and magnetic design of the WET-HET were performed using the finite element method magnetics (FEMM) software package and are discussed in Schwertheim et al. [23]. The WET-HET is considerably smaller than traditional thrusters of a similar power class, making thermal management difficult. The smaller volume of the ionization channel increases the density of the neutral gas to promote ionization. The model suggests radiative cooling has proved sufficient for powers up to approximately $1.5 \mathrm{~kW}$. For higher powers, optional water-cooling elements 
can be attached. The WET-HET is shown in Fig. 1. The thruster ionization channel is the volume between two concentric alumina tubes and truncated by a copper anode with a stainless steel cap. The magnetic field is generated by a single copper coil and shaped with soft iron components both inside and outside of the channel.

Previous studies utilized a zero-dimensional kinematic Boltzmann solver to simulate how oxygen molecules evolve over time in an environment such as a HET [22]. The results showed that the ionization fraction quickly rises to its maximum, and remains somewhat stable. This population starts primarily as diatomic ions $\left(\mathrm{O}_{2}^{+}\right)$, but once established, it quickly shifts such that the majority of the ions are monoatomic $\left(\mathrm{O}^{+}\right)$. In the context of a HET, a deeper channel results in a longer residence time for the propellant. This suggests that for the WET-HET, there is an optimal channel depth at which the ionization fraction is maximum. A deeper channel than this would result in a longer residence time, thus a greater ratio of $\mathrm{O}^{+}: \mathrm{O}_{2}^{+}$, and ultimately a lower thrust efficiency. The dissociation of the oxygen molecule is something that the PlasmaSim 0D code can not currently simulate. We plan instead to optimize this in the laboratory. The WET-HET has been designed so that the copper anode (number 10 in Fig. 1) can be removed and exchanged for longer or shorter sections, effectively making the thruster channel shallower or deeper. For this test the channel was 45 $\mathrm{mm}$ deep, which is the anode shown in Fig. 1.

The outer magnetic pole of the WET-HET is a stack of 10 soft iron rings (number 3 in Fig. 1). By physically raising a number of these rings up or down, the magnetic field topology of the thruster changes significantly. When a ring is raised a greater fraction of the thruster channel is exposed to the radial magnetic field, such that the magnetic thickness is increased. FEMM simulations of five of the ten possible magnetic topologies are shown in Fig. 2. Increasing the magnetic thickness is expected to increase the axial thickness of the Hall current, promoting electron-neutral collisions in an attempt to compensate for the smaller ionization cross section of oxygen. This is explained in further detail in Schwertheim et al. [23]. For the current study the thruster was configured in the 0 rings raised position, which we call the default position, and is the one shown in Fig. 1. This position represents the minimum magnetic thickness setting, and is the most similar to a traditional xenon hall effect thruster. The magnetic flux predicted by the FEMM magnetic model in this configuration is shown in Fig. 3 for different magnetic circuit currents. The circle points on the plot are the experimental flux densities measured with a magnetic probe.

It is important to note that the channel depth, and the magnetic topology can be changed independently of one another. This is in part due to the anode being constructed of copper and a stainless steel of low magnetic permeability, such that the shifting of the anode has little effect on the magnetic field. All the magnetic topologies shown in Fig. 2 can be achieved with the anode face positioned anywhere between -60 and $-35 \mathrm{~mm}$ from the exit plane. Changing the anode location changes the effective depth of the physical channel, but has no impact on the magnetic topology. For this test 0 rings were raised, and the anode face was kept at $-45 \mathrm{~mm}$, as can be seen in Fig. 3 .

Erosion is often a lifetime limiting factor of HETs. The oxygen plasma generated by the WET-HET is expected to be considerably more aggressive than a typical xenon plasma. Given that this thruster is designed as a proof of concept, we have chosen materials typical of conventional HETs, such as stainless steels and alumina. Assessing the degradation of these components will enable us to compare the erosion of the WET-HET to that of a traditional thruster.

\section{Experimental design}

This was the first thrust measurement test campaign of the WET-HET, and was conducted in the Boltzmann vacuum facility within the Imperial Plasma Propulsion Laboratory. A sensitivity analysis was performed to test how the thruster responds to a change in power, mass flow rate and magnetic field strength. For this test, the thruster channel depth and magnetic field configuration were not changed. These results were compared to the results of PlasmaSim OD at a range of electron temperatures to asses the strengths and weaknesses of the model.

All PlasmaSim 0D simulations were run for $1 \mathrm{~ms}$ of simulation time, with time steps of $0.1 \mu \mathrm{s}$ and 34000 superparticles. The first $0.1 \mathrm{~ms}$ of simulation time was excluded for the calculation of average values to allow the plasma to reach steady state. The simulation domain was the same dimensions as the internal channel dimensions of the WET-HET: $45 \mathrm{~mm}$ deep, $5 \mathrm{~mm}$ wide, and an average circumference of $62.8 \mathrm{~mm}$. Instead of modeling a cathode, the electrons are directly introduced at the exit plane. This means the cathode propellant choice has no impact on the simulation. Different simulations were run for electron temperatures in a Boltzmann distribution about $10 \mathrm{eV}, 11.5 \mathrm{eV}$ and $16 \mathrm{eV}$. For all simulations, the ion were distributed about $0.1 \mathrm{eV}$.

The WET-HET is optimized for operation between 1 and $2 \mathrm{~kW}$. The only available power supplies for this test campaign were not able to generate sufficient current for powers above approximately $1.2 \mathrm{~kW}$.

The Boltzmann facility has a $1.5 \mathrm{~m}$ diameter by $2 \mathrm{~m}$ long main chamber, with a $0.75 \mathrm{~m}$ diameter by $1.5 \mathrm{~m}$ long loadlock hatch. The facility is pumped by a roughing pump, two Leybold turbomolecular pumps each with a pumping speed of $2200 \mathrm{~L} / \mathrm{s}$ and a Leybold cryopanel at 15,000 L/s xenon.

To reduce the complexity of the test an electrolyser was not used. Zero grade oxygen was supplied to the thruster 
Fig. 1 WET-HET (top) with cross section (middle) and material description (bottom). The lower section of the top photograph shows the optional watercooling elements installed on the thruster, which are not present on the cross section.

These were not used in this test
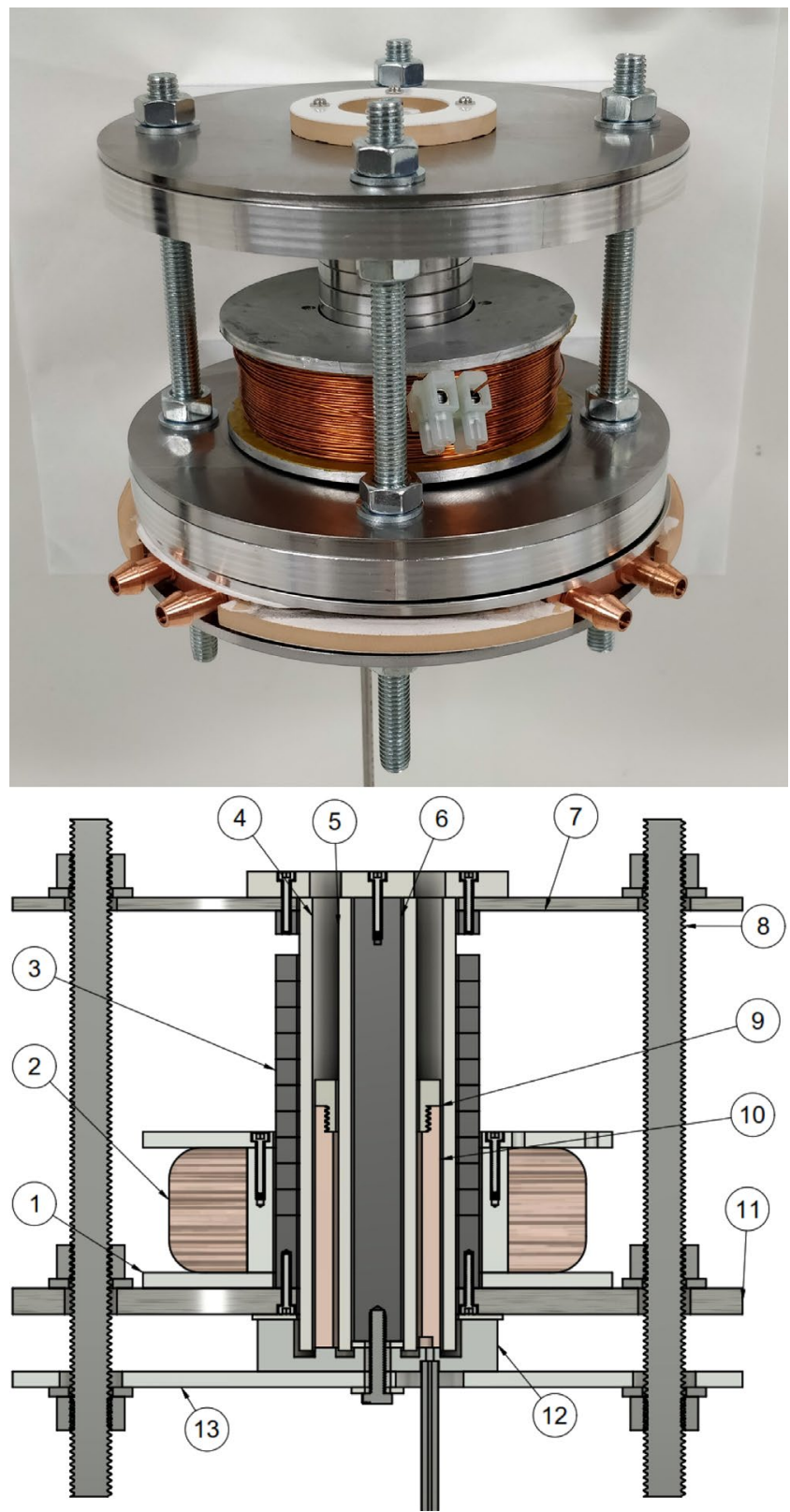

\begin{tabular}{|c|c|c|}
\hline No. & Name & Material \\
\hline 1 & Coil reel & Aluminium \\
\hline 2 & Magnet coil & Copper \\
\hline 3 & Outer pole stack & Soft Iron \\
\hline 4 & Outer channel wall & Alumina \\
\hline 5 & Inner channel wall & Alumina \\
\hline 6 & Inner pole & Soft Iron \\
\hline 7 & Front plate & Stainless steel \\
\hline 8 & Threaded rod structure & Stainless steel \\
\hline 9 & Anode cap & Stainless steel \\
\hline 10 & Anode & Copper \\
\hline 11 & Back plate & Stainless steel \\
\hline 12 & Tail cap & Stainless steel \\
\hline 13 & Radiator & Aluminium \\
\hline
\end{tabular}


Fig. 2 FEMM simulations of radial magnetic flux densities along the centre of the WETHET channel for five possible magnetic configurations. For this campaign only the 0 rings raised configuration was tested

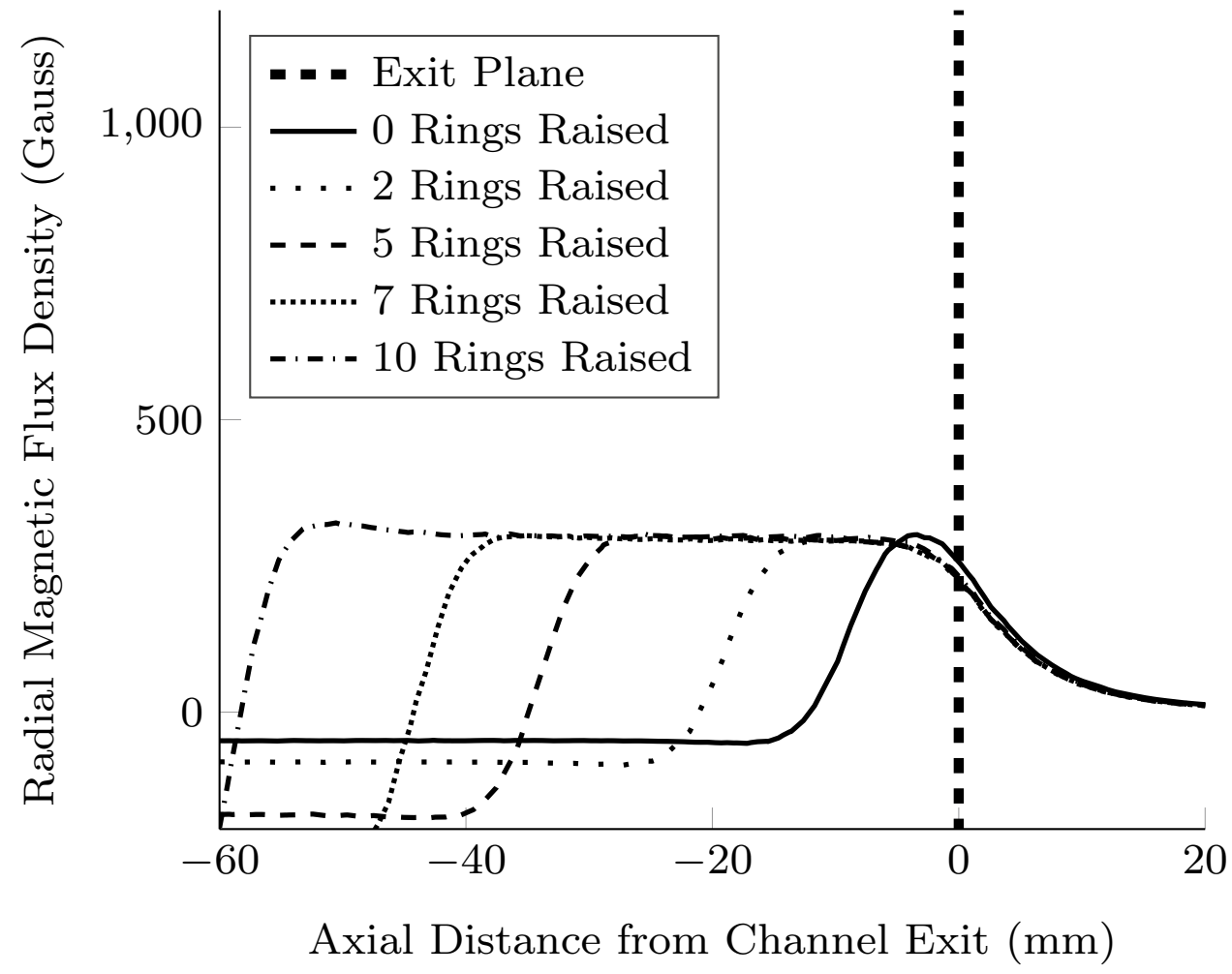

Fig. 3 The radial magnetic flux density along the center of the channel at different magnetic circuit currents with the rings in the 0 raised position. The lines show the results of the FEMM magnetic simulations. The circles show the experimentally measured values

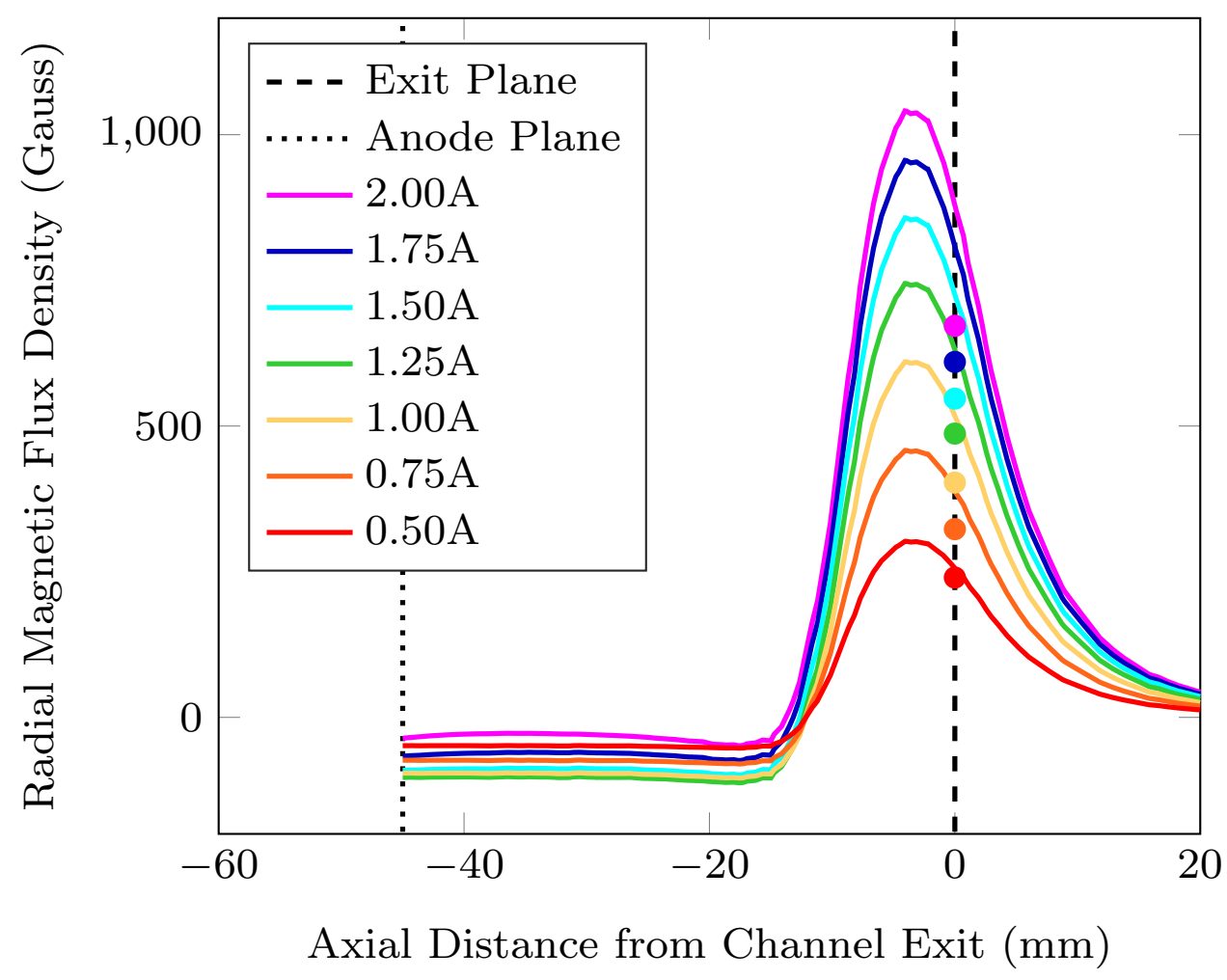

The ultimate goal of this project is to demonstrate the synergy between a thruster operating on oxygen, and a $\mathrm{LaB}_{6}$ cathode operating on hydrogen. However, for this test 
campaign the only available cathode available to us was a filament plasma bridge neutralizer [28]. In this type of cathode electrons are generated by thermionic emission of a tungsten filament which is directly heated by a DC current. A gas is injected into the cathode so that a plasma bridge can be established between the grounded filament, and a surrounding keeper electrode which is biased to $100 \mathrm{~V}$. The plasma can then be drawn out of the device by the thruster anode. The benefits of such a filament plasma bridge neutralizer over traditional emitter cathodes such as $\mathrm{LaB}_{6}$ cathodes are that they are cheap and simple to build and maintain. However, the filaments need to be replaced after several hours of operation, making them inappropriate for space missions.

We operated the filament plasma bridge neutralizer on 15 sccm research grade krypton for this test in place of hydrogen. The main focus of this campaign was the first characterization of the WET-HET device. Krypton was selected as the working gas for the cathode primarily due to health and safety constraints of our laboratory, which does not allow for a compressed hydrogen gas cylinder to be used in proximity to our oxygen feed system for the anode. A secondary consideration was a higher chamber pressure that would result from using hydrogen propellant, since the vacuum facility relies on a cyropanel as the primary pumping method.

The center of the cathode orifice was positioned $58 \mathrm{~mm}$ in front of the exit plane and $61 \mathrm{~mm}$ below the central axis of the thruster. The cathode was also tilted slightly less than vertical, such that the axis of the cathode made an angle of $82.5^{\circ}$ with the axis of the thruster. The cathode structure and the thruster structure are in electrical contact via the central platform of the thrust balance. This platform is allowed to electrically float.

\subsection{Thrust balance}

The thrust of the WET-HET was evaluated using a hanging pendulum style thrust balance which has been developed in house for direct thrust measurements between 1 and 100 $\mathrm{mN}$. The thruster and cathode are secured to a central platform which is suspended from the structure of the balance by four parallel linkages, each containing two stainless steel flexures. The central platform is the hanging pendulum element of the balance. This is shown in Fig. 4.

When firing, the thruster displaces the central platform with respect to the stationary base plate. A Micro-Eplison optoNCDT 1750-10 optical laser triangulation sensor measures this displacement with sub-micron precision 7500 times a second.

The thrust balance is calibrated using a AVM12-6.4 voice coil actuator (VCA) manufactured by Akribis Systems. We use a VCA because when paired with a precision sourcemeter the two can produce a very precise and repeatable non-contact force. First the VCA is characterized by
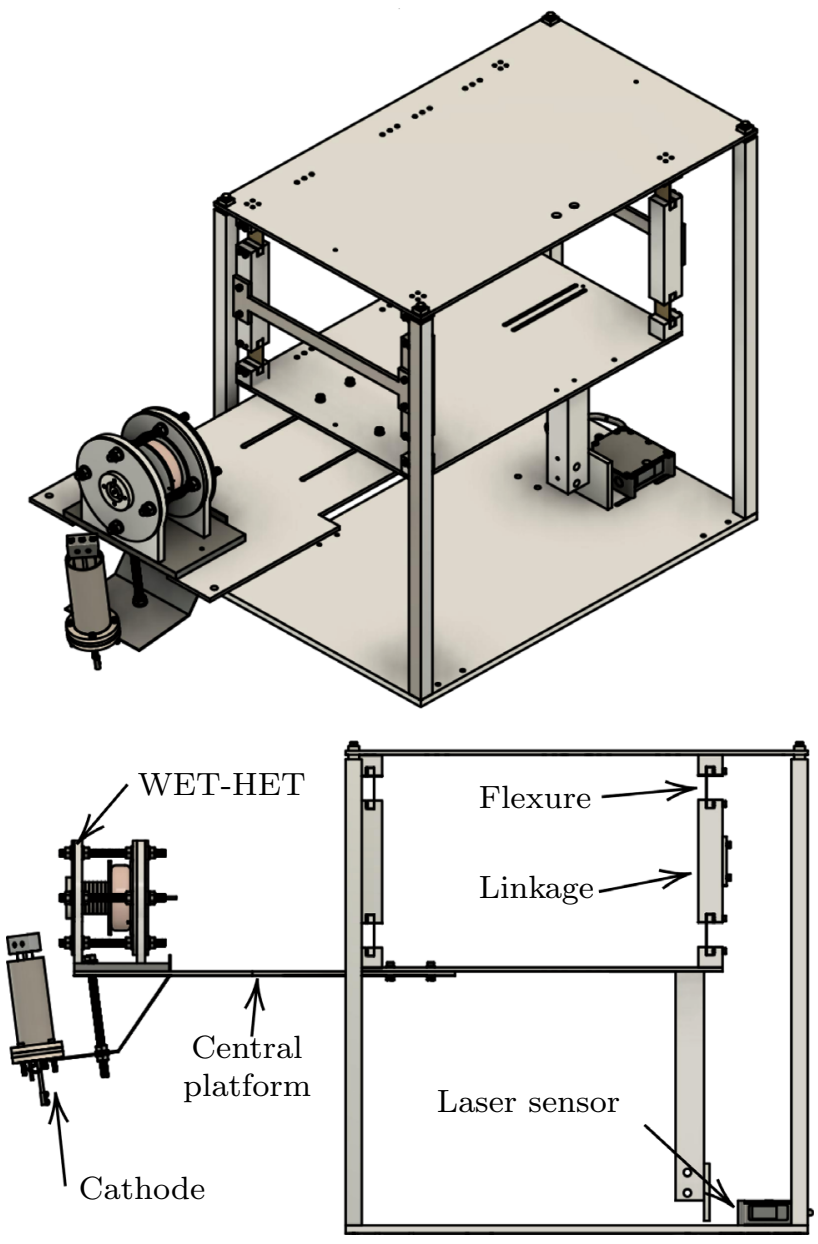

Fig. 4 Simplified isometric (top) and side (bottom) view of the balance with the WET-HET and cathode mounted. The direction of thrust is to the right in the bottom figure

measuring the force it produces at a range of currents on a high precision microbalance. This microbalance itself has been calibrated to the National Measurement Institute standards. Shortly before we evacuate our vacuum facility we position the VCA such that a range of precise non-contact forces are imposed on the moving central platform of the thrust balance. This force is representative of a thrust produced by the thruster. The laser triangulation sensor is used to accurately measure the relationship between known force and balance displacement. This effectively allows us to use the VCA as a link in a calibration chain that ties our balance to the independently calibrated microbalance. The balance displacement as a response to these known forces ranging from 0.39 to $172 \mathrm{mN}$ is plotted in Fig. 5. The gradient of this line is found using standard curve fitting tools and is called the sensitivity $S_{\text {cal }}$. With the sensitivity accurately measured, we can now calculate an accurate thrust measurement from a measured displacement. For this campaign, the sensitivity of the balance was 
Fig. 5 The response of the thrust balance to the known forces produced by the VCA with the standardized residuals

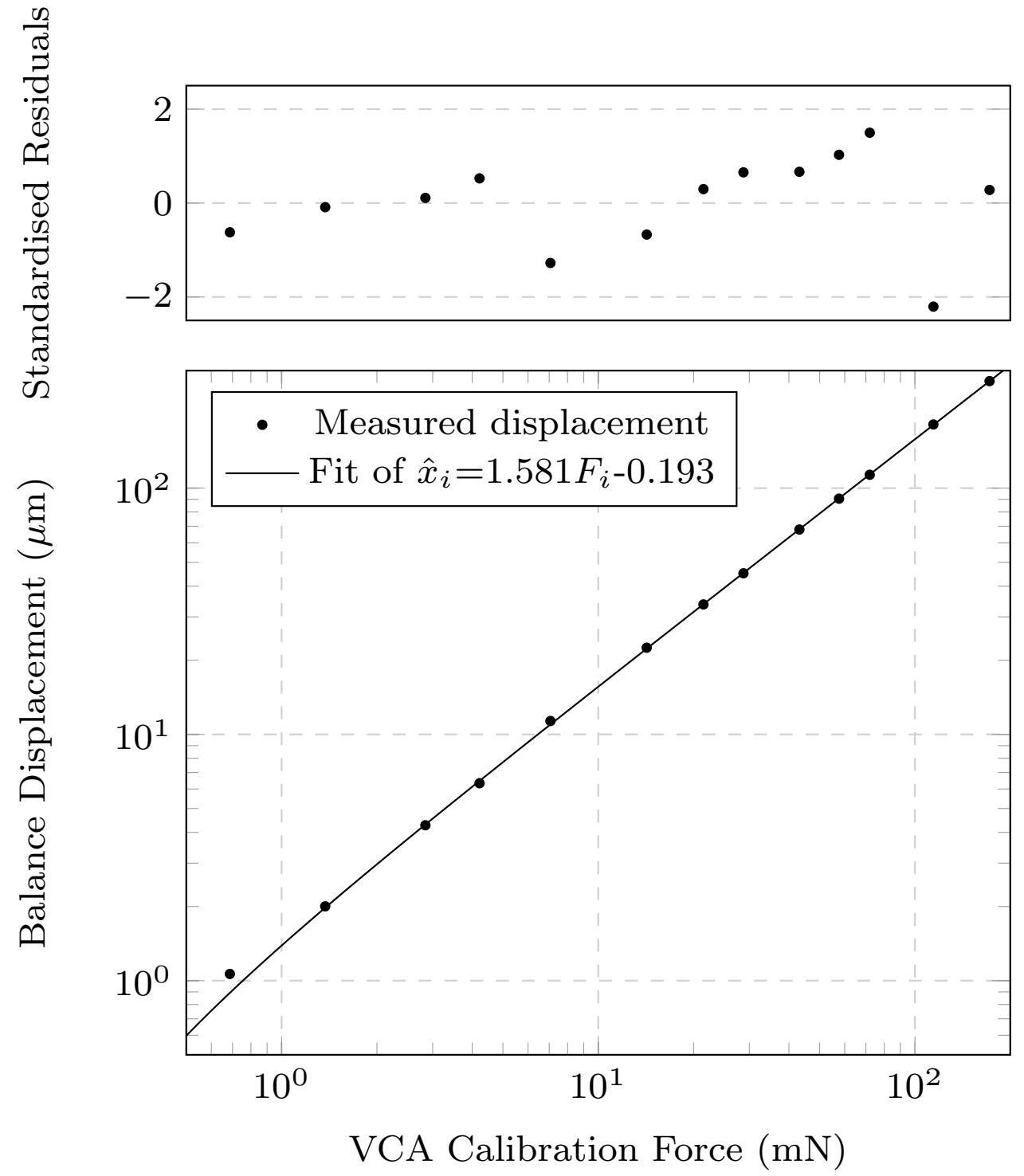

$\frac{\sigma_{F_{t}}}{F_{t}}=\sqrt{\left(\frac{\sigma_{x}}{x}\right)^{2}+\left(\frac{\sigma_{S_{\text {cal }}}}{S_{\text {cal }}}\right)^{2}}$.

found to be $S_{\text {cal }}=1.581 \pm 0.0015 \mu \mathrm{m} / \mathrm{mN}$. The standardized residuals $R_{s}$ shown in the top of Fig. 5 are used to asses the quality of fit. They are calculated by:

$R_{s}=\left(\hat{x}_{i}-x_{i}\right) / s_{x}$

where $x_{i}$ is the measured balance displacement for calibration force $F_{i}$, and $\hat{x}_{i}$ is the predicted balance displacement for calibration force $F_{i}$ found from $\hat{x}_{i}=S_{\text {cal }} F_{i}$. Here $s_{x}$ is the estimate of the standard deviation of the random disturbances to the balance. As a rule of thumb, standardized residuals distributed uniformly between -2 and 2 are considered indicative of a good fit.

The post processing of the balance data was performed using the outline set by Polk et al. [17]. For a thrust measurement $F_{t}$ generated by a measured displacement $x$, the thrust measurement has an uncertainty $\sigma_{F_{t}}$ given by:
This equation is adapted from Eq. (31) of Polk et al. [17]. Here $\sigma_{x}$ is the standard deviation of the random disturbances to the balance, and $\sigma_{S_{\text {cal }}}$ is the standard deviation of the thrust balance sensitivity. These parameters are derived following the method set out in Polk et al. [17].

The electromagnetic interference that can be produced by EP testing has the ability to induce currents in the VCA circuit. These currents have been shown to produce erroneous thrust signals which can be extremely difficult to discern from real thrust [19]. To eliminate this source of uncertainty we remove the VCA subsystem from the balance prior to testing. To ensure the calibration does not change throughout the test, a servo motor is used to 
perturb a test mass attached to the moving platform of the balance.

The final vacuum set-up of the thruster, cathode and thrust balance can be seen in Fig. 6. All electrical and propellant lines that pass from the balance structure to the moving platform where connected using terminals that were as thin and flexible as possible to reduce additional stiffness of the flexures. These lines were connected in wide arcs running perpendicular to the thrust axis between the moving and stationary portions of the thrust balance such that any thermal expansion would lead to off-axis forces which would not be registered as thrust. The effect of spurious electrostatic forces induced by the thruster electrical wiring was tested. This was done by raising the voltage anode as high as possible, and operating the magnetic current while observing the laser displacement sensor. Neither of these actions caused a thrust discernible from the noise floor.

A cold gas flow test was undertaken to identify the influence of pressurizing the propellant feed-lines on the measured thrust. This was done by flowing gas through the thruster and anode while both were unpowered and under vacuum. The observed thrust was expected to be consistent with cold gas thrust operating on oxygen without a nozzle: around $40-60 \mathrm{~s}$ of $I_{\mathrm{sp}}$. We measured $0.76 \pm 0.17 \mathrm{mN}$ of thrust for a mass flow rate of $1.45 \mathrm{mg} / \mathrm{s}$, which results in an $I_{\mathrm{sp}}$ of $53 \pm 12 \mathrm{~s}$. These values suggest the propellant line pressurization is not leading to erroneous thrust measurements.

\section{Results}

The WET-HET has proven to ignite easily on oxygen mass flow rates down to $1.2 \mathrm{mg} / \mathrm{s}$. To sustain the discharge at magnetic circuit currents over $1 \mathrm{~A}$ required a mass flow rate of at least $1.45 \mathrm{mg} / \mathrm{s}$. The WET-HET is shown both with and without an applied magnetic field in Fig. 7. A very distinctive color change from green-white to pink-white is seen when the magnetic field is applied. This visual observation suggests an increase in the temperature of the electron population within the channel leading to a change in the neutral and ion excitation states and transitions. However, no spectroscopic analysis has been performed at this stage.

Figure 8 shows how the thrust, $I_{\mathrm{sp}}$, and anode thrust efficiency of the WET-HET changes with power. The mass flow rate and magnetic circuit current was $1.45 \mathrm{mg} / \mathrm{s}$ and 1.5 A respectively for all these data. The thrust and $I_{\mathrm{sp}}$
Fig. 6 The front on view of the WET-HET and cathode mounted to the thrust balance in the Boltzmann vacuum facility

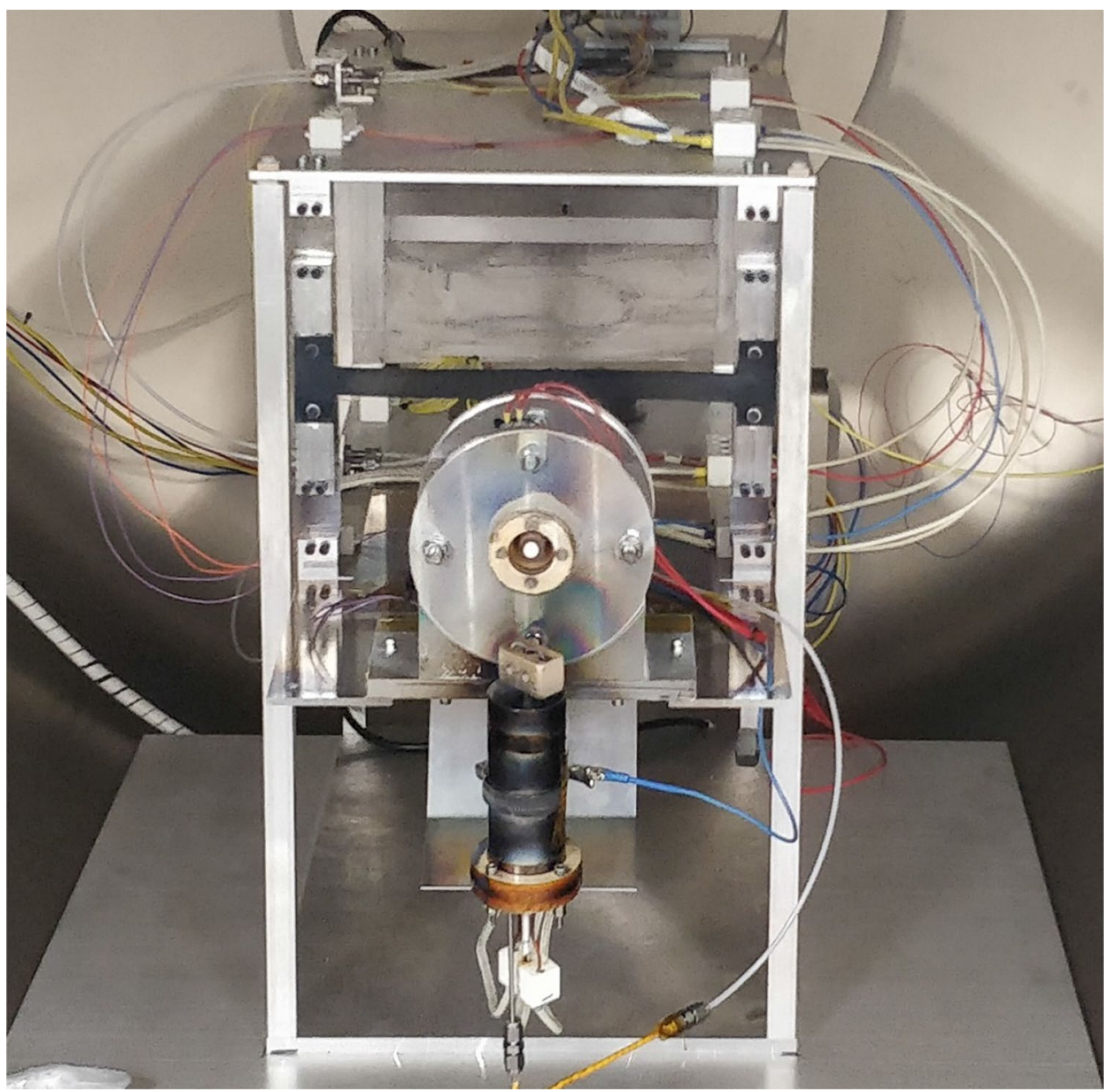



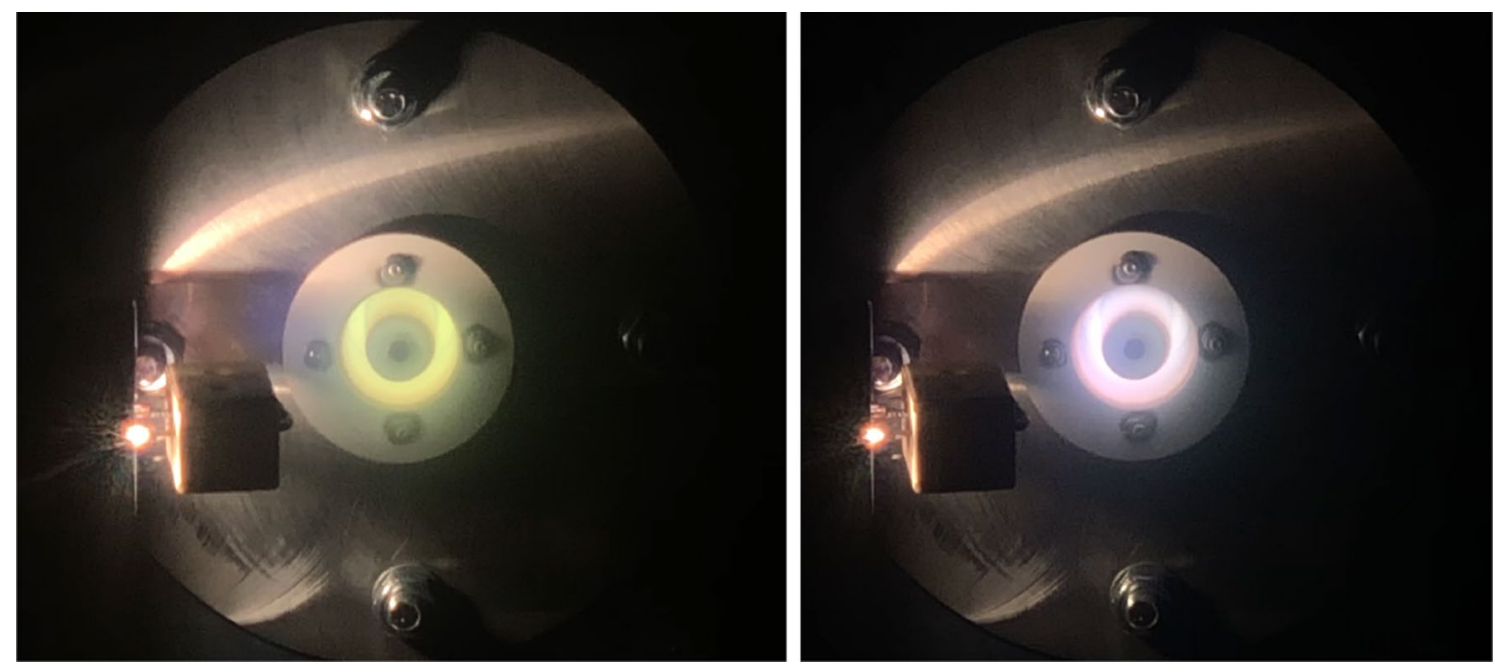

Fig. 7 The WET-HET operating on $1.32 \mathrm{mg} / \mathrm{s}$ and 2.5 A discharge current. With no current applied to the magnetic circuit (left) and with $0.5 \mathrm{~A}$ of current applied to the magnetic circuit (right). Please

are shown clearly to increase linearly with power, with no obvious plateau. This same trend is reflected in the PlasmaSim results for all electron temperatures, however at considerably greater values. The trends shown in these simulations appear to are surprisingly consistent across different electron temperatures. We define anode thrust efficiency $\eta_{T}$ as:

$\eta_{T}=\frac{T^{2}}{2 \dot{m}_{a} P_{a}}$

where $T$ is thrust, $\dot{m}_{a}$ is the anode mass flow rate and $P_{a}$ is the anode power. The anode thrust efficiency derived from the experimental data also shows to linearly increase with power. The anode thrust efficiency predicted by the simulation greatly overestimates the data. The PlasmaSim 0D simulations at the two lower electron temperatures appear to have reached a maximum, whereas the $16 \mathrm{eV}$ results increase linearly over the entire range. Here the $16 \mathrm{eV}$ trend is the only one that reflects the experimental data. Only those simulations that were able to maintain a stable discharge are plotted in Fig. 8. For this reason the $10 \mathrm{eV}$ data are only available for low powers.

We can assume some of the discrepancy between the experimental data and the PlasmaSim data can be attributed to the fact that the simulation is not accounting for several energy sinks. The power lost to the electrical excitation or the vibration, rotation, or breaking of the covalent bond is not modeled in the current version of PlasmaSim.

The three plots of Fig. 9 show the dependency of thrust, $I_{\text {sp }}$ and efficiency on mass flow rate. Here power was kept constant at $600 \mathrm{~W}$ and the magnetic circuit current was 0.5 note the cathode was positioned differently at this stage of test, but was moved to the position described in the text and shown in Fig. 6 before any thrust measurements were taken

A. Note that these data were taken at a lower magnetic circuit current than those in Fig. 8 because low mass flow rates are unable to sustain the higher magnetic field strengths. In the experimental data, an increase in mass flow rate is shown to lead to a higher thrust at the expense of $I_{\mathrm{sp}}$. The anode thrust efficiency is constant over the range of mass flow rates surveyed. We again see PlasmaSim predicting a similar trend but at greater absolute values for both thrust and $I_{\mathrm{sp}}$ for all electron temperatures. For the simulations of anode thrust efficiency there is little agreement across different electron temperatures. The $10 \mathrm{eV}$ trend appears to be increasing, yet this plasma was unsustainable at mass flow rates below $1.5 \mathrm{mg} / \mathrm{s}$, and has been omitted. When the electron temperature is set to $11.5 \mathrm{eV}$, there appears to be a maximum anode thrust efficiency near $1.4 \mathrm{mg} / \mathrm{s}$. The $16 \mathrm{eV}$ trend is consistently decreasing. For anode thrust efficiency, none of the simulation trends are reflective of our experimental observations.

The sensitivity of the WET-HET to a change in magnetic circuit current is shown in Fig. 10. For these measurements, the power was fixed at $750 \mathrm{~W}$, and the mass flow rate was constant at $1.45 \mathrm{mg} / \mathrm{s}$. The thrust and specific impulse both rise to an optimum near $1 \mathrm{~A}$, after which they drop off. The lower plot shows that above 1 A the discharge voltage continues to increase although thrust does not. One possible explanation for this is that the increased magnetic field reduces the size of the ion gyroradius so that it becomes comparable to the thruster dimensions. The gyroradius $r_{g}$ of the ions is given by: 
Fig. 8 Thrust, specific impulse and thrust efficiency of the WET-HET operating on 1.45 $\mathrm{mg} / \mathrm{s}$ of anode mass flow rate and 1.5 A magnetic circuit current. Also shown are the results of PlasmaSim results at different electron temperatures
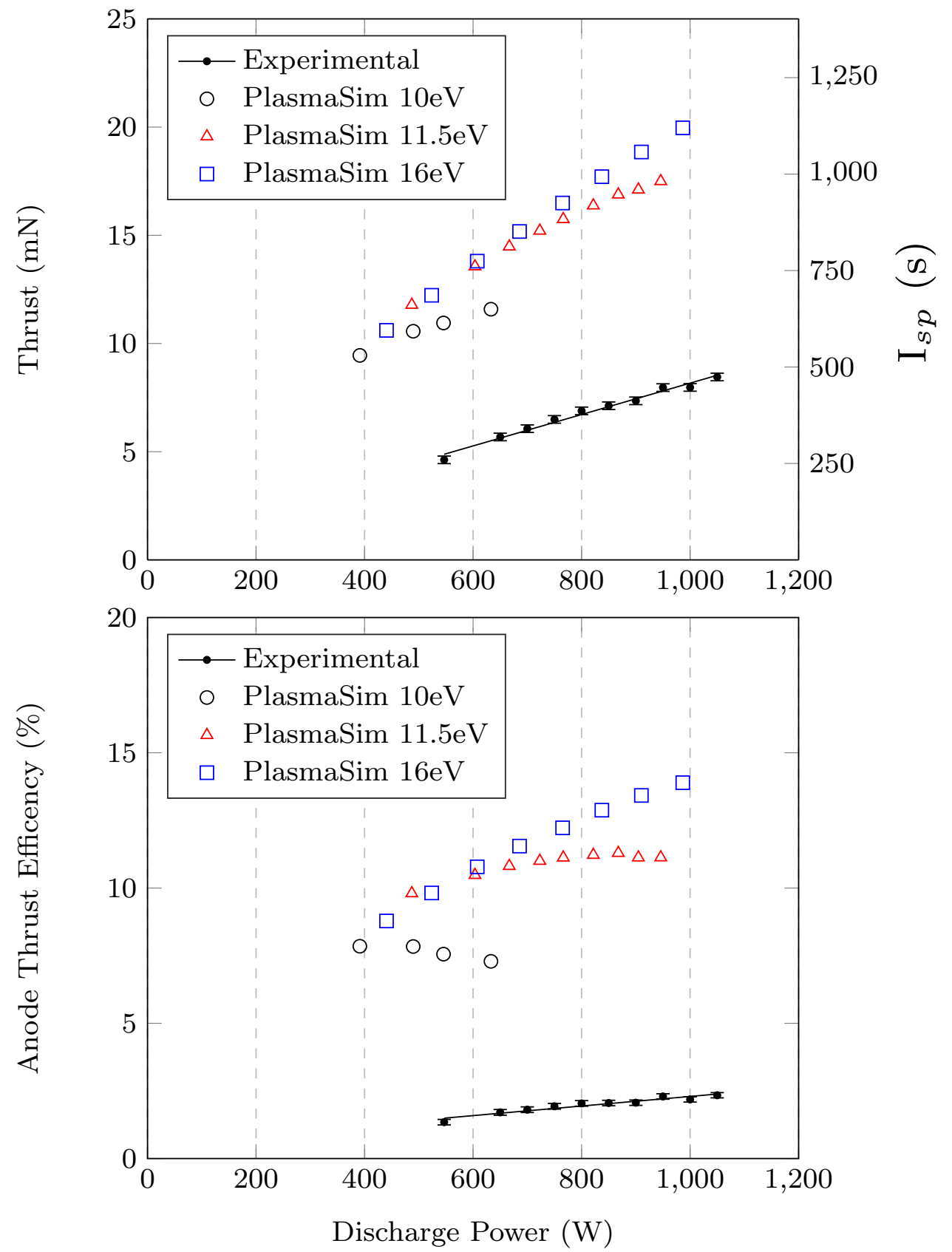

$r_{g}=\frac{m v_{\perp}}{|q| B}$

measured at $403 \mathrm{G}$. From the experimental data we see this results in a discharge voltage of $191 \mathrm{~V}$. If we substitute these values into Eq. (4) we derive a possible ion gyroradius range from 6.4 to $279 \mathrm{~mm}$ for $\mathrm{O}_{2}^{+}$ions, and from 4.5 to $197 \mathrm{~mm}$ for $\mathrm{O}^{+}$ions. These gyroradii are of the same order of magnitude as the channel length and width of the WET-HET. This suggest that a portion of the ion population will be deflected or even trapped by the magnetic field, and thus contribute to less thrust. This could possibly account for why greater magnetic circuit currents can generate a greater discharge voltage, yet not lead to a higher acceleration of ions, as seen by a lower thrust and $I_{\mathrm{sp}}$. 
Fig. 9 Mass flow rate sensitivity for thrust, $I_{\mathrm{sp}}$ and thrust efficiency for operation at 600 $\mathrm{W}$ and $0.5 \mathrm{~A}$ magnetic circuit current. Also shown are the results of PlasmaSim results at different electron temperatures
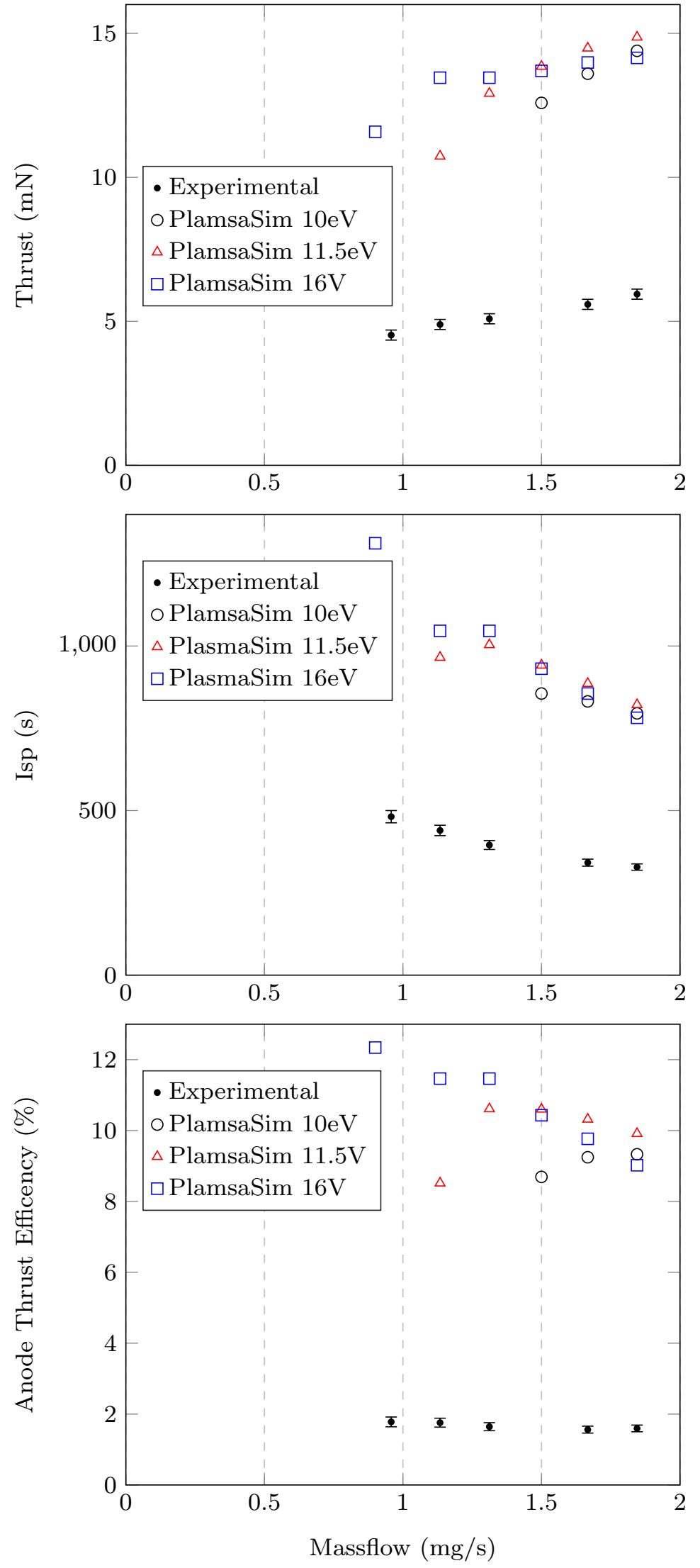
Fig. 10 Magnetic circuit current sensitivity of the WETHET at $750 \mathrm{~W}$ and $1.45 \mathrm{mg} / \mathrm{s}$ mass flow rate
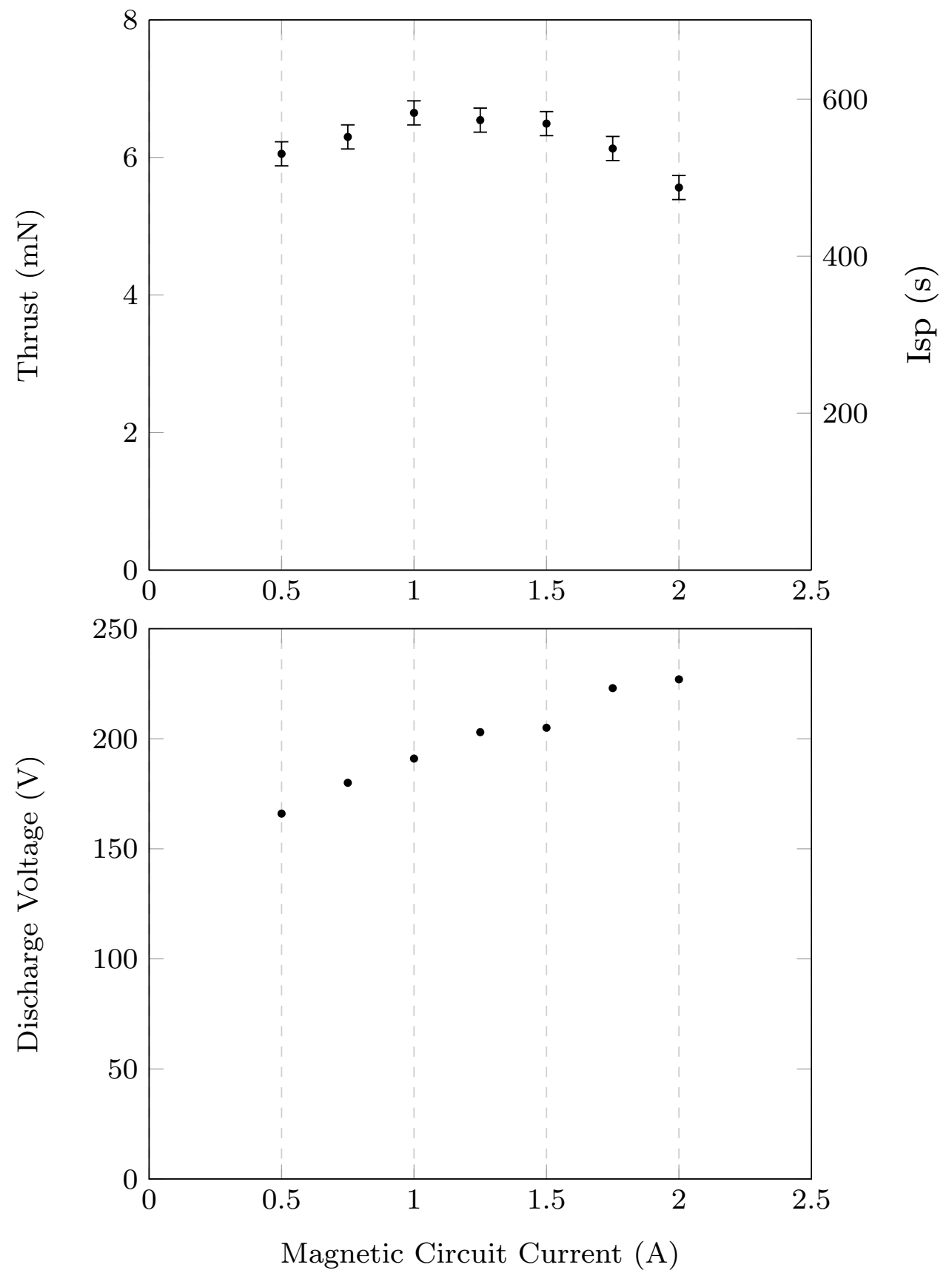

The PlasmaSim 0D code has shown to greatly over estimate WET-HET performance for all electron temperatures simulated. We assume that this is due to the generalizations we were forced to make to simplify the original 3D code. These include imposing a constant electric field along the channel, enforcing a uniform electron temperature distribution and omitting magnetic effects altogether. The code also fails to simulate the energy lost to electrical excitation, dissociation, vibration and rotation of the molecule. Even with these shortcomings, the model appears to accurately predict many of the trends we see in the experimental data. PlasmaSim OD has successfully predicted how the thrust and $I_{\mathrm{sp}}$ respond to a change in power and mass flow rate. The model is less successful at predicting how thrust efficiency responds to these changes. In the majority of cases, the PlasmaSim OD trends were consistent across the three electron temperatures surveyed. Exceptions to this was the sensitivity of the anode thrust efficiency to mass flow rate, where the trends varied between the three electron temperatures, none of which were found to match the experimentally measured anode efficiency. The discrepancy between the experimental data and those produced by PlasmaSim 0D is too great for us to accurately describe which electron temperature is most 
representative of those seen in the laboratory. Future work will aim to experimentally measure the axial distribution of electron temperature through direct plasma probe measurements.

\section{Discussion and future work}

Our first performance measurements of the WET-HET device have indicated an anode thrust efficiency that is far lower than for conventional HETs operating on xenon. However, there is a large range of experimentally tunable parameters that have yet to be investigated, including the channel depth and magnetic field thickness, that are likely to strongly influence the thruster performance. At $1050 \mathrm{~W}$ of input power the WET-HET produces $8.45 \pm 0.18 \mathrm{mN}$ of thrust at $593 \pm 12 \mathrm{~s}$ of $I_{\mathrm{sp}}$, which results in an anode thrust efficiency of $2.34 \pm 0.10 \%$. In comparison, a SPT-100 operating at the same power will produce $64.1 \mathrm{mN}$ at $1480 \mathrm{~s}$ of $I_{\mathrm{sp}}$, which results in an efficiency of $46.92 \%$ [21]. Even with the discussed system level benefits of the WET-HET, namely lower propellant price, propellant sharing potential, and ISRU possibilities, the current level of anode thrust efficiency would severely limit the commercial viability of this technology. Therefore, future experimental efforts will be aimed at increasing the thrust efficiency of the device.

The WET-HET is the very first prototype of a HET optimized for oxygen operation. Although the preliminary performance leave much to be desired, this could yet improve due to the thruster having a rich trade space of tunable parameters we are yet to explore. The most promising avenues for thruster optimization include tailoring the channel depth and magnetic field thickness, which will be the focus of the next experimental campaigns. We will also investigate higher levels of power, up to the $1.5 \mathrm{~kW}$ design target and beyond, to determine where the thrust efficiency plateaus. Additionally, the independent variables of mass flow rate and magnetic field strength will be surveyed across a wide range.

We have shown that the gyroradius of the oxygen ions are comparable to some of the features of the discharge channel. Evidence of this is a lower thrust at high magnetic circuits currents as we have shown. This is generally not a problem with xenon HETs at these magnetic field strengths, due to the much larger gyroradius of the heavy xenon ions. This further suggests that an oxygen HET such as the WET-HET may benefit from a deeper ionization channel, which has a magnetic topology with a thicker magnetic region, but of a lower magnitude. Tests with different magnetic typologies will investigate this.

Future tests will examine the influence of switching the cathode feed gas to hydrogen, in its intended configuration of an electrolysis fed propellant delivery system. This will further constrain the flow rate to the cathode as a fixed fraction of the flow rate to the anode based on mass ratio of hydrogen and oxygen within water. Plasma probe diagnostics are also planned to measure the electron temperature distribution within the channel of the WET-HET, which has been found to be a critical parameter for the thruster modeling. Longer tests will also shed more light on how quickly the reactive oxygen plasma erodes different components of the thruster, as this has large implications on the lifetime of potential missions.

\section{Conclusion}

We suggest that a HET could be modified to operate on the products of in situ water electrolysis. The issue of cathode poisoning can be mitigated by supplying the anode with oxygen, and the cathode with the produced hydrogen. Not only would such a system benefit from the low price, high storability and ubiquity of water, but synergy with a water electrolysis chemical propulsion system suggests the opportunity for a shared propellant chemical-electrical multimode propulsion system.

The water electrolysis Hall-effect thruster (WET-HET) has been designed to test this concept. The thruster channel dimensions have been optimized for oxygen operation using a OD version of a PIC code called PlasmaSim. Both the channel depth and magnetic topology of the thruster can be modified in the laboratory.

A hanging pendulum style thrust balance was used to monitor how the thrust, $I_{\mathrm{sp}}$, and anode thrust efficiency of the WET-HET change with power, mass flow rate, and magnetic circuit current. Thrust, $I_{\mathrm{sp}}$ and thrust efficiency were shown to rise steadily for powers between 550 and $1050 \mathrm{~W}$. Increasing the mass flow rate from 0.96 to $1.85 \mathrm{mg} / \mathrm{s}$ resulted in an increasing thrust, decreasing $I_{\mathrm{sp}}$ but had little impact on the thrust efficiency. Both thrust and $I_{\mathrm{sp}}$ are optimum when $1 \mathrm{~A}$ of current passes through the magnetic circuit, which results in a flux density of approximately $403 \mathrm{G}$ at the exit plane. Greater magnetic field strengths than this increase discharge voltage, but this rise is not reflected in thrust or $I_{\mathrm{sp}}$. We suggest this may be due to interactions of the oxygen ions with the magnetic field at higher field strengths leading to curvature or capture of the outgoing ion trajectories, which ultimately reduces thrust.

When compared to the experimental data, the $0 \mathrm{D}$ version of PlasmaSim has shown to greatly overestimate thrust, $I_{\mathrm{sp}}$ and anode thrust efficiency for all electron temperatures surveyed. We assume that this is due to the internal energy of the molecule not being accounted for, and the dissociating to atomic oxygen not being modeled. Although the values of thrust and $I_{\mathrm{sp}}$ produced by PlasmaSim differ greatly from 
the experimental data, the trends they show generally appear to be consistent.

Future sensitivity studies will be conducted to determine how changing the channel depth and magnetic field topology of the WET-HET will impact thruster performance, in the hope of making it more commercially competitive. Further testing will assess thruster performance at powers up to 2 $\mathrm{kW}$. Research efforts will also target the shortcomings of PlasmaSim to better model the internal energy state of the oxygen molecule in the hopes that a more predictive model can be produced.

Open Access This article is licensed under a Creative Commons Attribution 4.0 International License, which permits use, sharing, adaptation, distribution and reproduction in any medium or format, as long as you give appropriate credit to the original author(s) and the source, provide a link to the Creative Commons licence, and indicate if changes were made. The images or other third party material in this article are included in the article's Creative Commons licence, unless indicated otherwise in a credit line to the material. If material is not included in the article's Creative Commons licence and your intended use is not permitted by statutory regulation or exceeds the permitted use, you will need to obtain permission directly from the copyright holder. To view a copy of this licence, visit http://creativecommons.org/licenses/by/4.0/.

\section{References}

1. Ahedo, E., Escobar, D.: Influence of design and operation parameters on hall thruster performances. J. Appl. Phys. 96(2), 983-992 (2004)

2. Avdienko, A., Malev, M.: Poisoning of lab6 cathodes. Vacuum 27(10-11), 583-588 (1977)

3. Bugrova, A., Desiatskov, A., Kharchevnikov, V., Morozov, A. Main features of physical processes in stationary plasma thrusters. In: 23rd International propulsion conference (1993)

4. Campbell, J.G., Stechman, R.C., Jr.: Water electrolysis propulsion system testing. Tech. rep, MARQUARDT CO VAN NUYS CA (1974)

5. Carrasquillo, R.: Iss eclss technology evolution for exploration. In: 43rd AIAA aerospace sciences meeting and exhibit, p. 337 (2005)

6. Croes, V., Lafleur, T., Bonaventura, Z., Bourdon, A., Chabert, P.: 2D particle-in-cell simulations of the electron drift instability and associated anomalous electron transport in hall-effect thrusters. Plasma Sources Sci. Technol. 26(3), 034001 (2017)

7. Gallagher, H.: Poisoning of lab6 cathodes. J. Appl. Phys. 40(1), 44-51 (1969)

8. Goebel, D., Hirooka, Y., Sketchley, T.: Large-area lanthanum hexaboride electron emitter. Rev. Sci. Instrum. 56(9), 1717-1722 (1985)

9. Goebel, D.M., Katz, I.: Fundamentals of electric propulsion: ion and Hall thrusters, vol. 1. John Wiley \& Sons, Hoboken (2008)

10. Itikawa, Y.: Cross sections for electron collisions with oxygen molecules. J. Phys. Chem. Ref. Data 38(1), 1-20 (2009)

11. James, K., Bodnar, M., Freedman, M., Osborne, L., Grist, R., Hoyt, R.: Hydros: high performance water-electrolysis propulsion for cubesats and microsats. Adv. Astronaut. Sci. Guid. Navig. Control 159, 2017-145 (2017)

12. Kieckhafer, A., King, L.B.: Energetics of propellant options for high-power hall thrusters. J. Propuls. Power 23(1), 21-26 (2007)

13. Linnell, J.A., Gallimore, A.D.: Krypton performance optimization in high-voltage hall thrusters. J. Propuls. Power 22(4), 921-925 (2006)

14. Martinez, D.R., Aanesland, A.: Development and testing of the npt30-i2 iodine ion thruster. In: 36th International electric propulsion conference, pp. 15-20 (2019)

15. Mirtich, M.J.: Resistojet propulsion for large spacecraft systems. In: 16th International electric propulsion conference (1982)

16. Muir, C., Knoll, A.: Catalytic combustion of hydrogen and oxygen for an electrolysis micro-propulsion system. J. Br. Interplanet. Soc. 72(1), 2-6 (2019)

17. Polk, J.E., Pancotti, A., Haag, T., King, S., Walker, M., Blakely, J., Ziemer, J.: Recommended practices in thrust measurements. Tech. rep, CALIFORNIA INST OF TECHNOLOGY PASADENA JET PROPULSION LAB (2013)

18. Potrivitu, G.C., Xu, L., Huang, S., Rohaizat, M., Xu, S.: Discharge mode transition in a krypton-fed 1 a-class lab6 cathode for lowpower hall thrusters for small satellites. J. Appl. Phys. 127(6), 064501 (2020)

19. Pottinger, S., Lamprou, D., Knoll, A., Lappas, V.: Impact of plasma noise on a direct thrust measurement system. Rev. Sci. Instrum. 83(3), 033504 (2012)

20. Rovey, J., Lyne, C.T., Mundahl, A.J., Rasmont, N., Glascock, M.S., Wainwright, M.J., Berg, S.P.: Review of chemical-electric multimode space propulsion. In: AIAA propulsion and energy 2019 forum, p. 4169 (2019)

21. Sankovic, J.M., Hamley, J.A., Haag, T.W.: Performance evaluation of the Russian SPT-100 thruster at NASA LeRC. In: 23rd International electric propulsion conference (1994)

22. Schwertheim, A., Knoll, A.: In situ utilization of water as a propellant for a next-generation plasma propulsion system. JBIS J. Br. Interplanet. Soc. 72(1), 7-11 (2019)

23. Schwertheim, A., Knoll, A.: The water electrolysis hall effect thruster (wet-het): Paving the way to dual mode chemical-electric water propulsion. In: 36th International electric propulsion conference, p. 259 (2019)

24. Stephan, K., Märk, T.: Absolute partial electron impact ionization cross sections of xe from threshold up to $180 \mathrm{ev}$. J. Chem. Phys. 81(7), 3116-3117 (1984)

25. Sturges, D., Oskam, H.: Hollow-cathode glow discharge in hydrogen and the noble gases. J. Appl. Phys. 37(6), 2405-2412 (1966)

26. Szabo, J., Tedrake, R., Kolencik, G., Pote, B.: Measurements of a krypton fed $1.5 \mathrm{kw}$ hall effect thruster with a centrally located cathode. In: The 35th international electric propulsion conference, Atlanta, Georgia, USA, IEPC-2017-26 (2017)

27. Thompson, S.J., Farnell, C.C., Farnell, S.C., Williams, D.D., Chandler, J.P., Williams, J.D.: Evaluation of iodine compatible cathode configurations. In: 36th International electric propulsion conference (2019)

28. Wolf, B.: Handbook of ion sources. CRC Press, Boca Raton (1995)

29. Zacny, K., Metzger, P., Luczek, K., Mantovani, J., Mueller, R.P., Spring, J.: The world is not enough (wine): Harvesting local resources for eternal exploration of space. In: AIAA SPACE 2016. aerospace research central (2016). https://doi. org/10.2514/6.2016-5279 